\title{
The Serum Angiotensinogen Concentration and Variants of the Angiotensinogen Gene in White and Black Children
}

Laura J. Bloem, Amita K. Manatunga, Duane A. Tewksbury, ${ }^{*}$ and J. Howard Pratt

Department of Medicine, Indiana University School of Medicine and the Veterans Administration Hospital, Indianapolis, Indiana 46202; and the *Marshfield Medical Research Foundation, Marshfield, Wisconsin 54449

\begin{abstract}
The T235 allele of the angiotensinogen gene $(A G T)$ has been associated with hypertension. Blood pressure increases faster over time in black children than in white children, and in adults hypertension is more prevalent in blacks. We sought evidence for a role for angiotensinogen to contribute to racial differences in blood pressure in a study of 148 white and 62 black normotensive children (mean age, 14.8 yr). The frequency of the T235 allele was 0.81 in blacks and 0.42 in whites $\left(\chi^{2}=77.3, P=0.0001\right)$. The mean angiotensinogen level was $19 \%$ higher in blacks than in whites ( $P$ $=0.0001$ for males, $P=0.004$ for females). Genotype was positively related to serum angiotensinogen in white children ( $P=0.0001$ for males, $P=0.004$ for females $)$, but a similar relationship was absent in blacks where the frequency of M235 may have been too low to discern an association. Longitudinal blood pressure (measured twice yearly) adjusted for body mass index showed a marginally significant relationship to the angiotensinogen level $(P=0.07)$. An independent relationship of serum angiotensinogen with body mass index $(P=0.0001)$ and race $(P=0.0003)$ was also observed. In summary, T235 was more frequent, and the level of angiotensinogen was higher in blacks than in whites. Such a racial difference in the renin-angiotensin system may contribute to the disparity in blood pressure levels of white and black young people. (J. Clin. Invest. 1995. 95:948-953.) Key words: angiotensinogen • angiotensinogen gene $\cdot$ race $\cdot$ children $\cdot$ blood pressure
\end{abstract}

\section{Introduction}

Angiotensinogen, a protein synthesized principally in the liver, interacts with renin to produce angiotensin I, the prohormone of angiotensin II (AII). ${ }^{1}$ AII promotes sodium retention and increases vascular resistance and is thus important for blood pressure regulation. The concentration of angiotensinogen is a rate-limiting factor in the generation of AII (1), and its circulat-

Address correspondence to J. Howard Pratt, MD, Indiana University Medical Center, 541 Clinical Dr., Indianapolis, IN 46202-5111. Phone: 317-274-0790; FAX: 317-274-7700.

Received for publication 6 April 1994 and in revised form 31 October 1994.

1. Abbreviations used in this paper: $\mathrm{AI}$, angiotensin I; AII, angiotensin II; $A G T$, the angiotensinogen gene; BMI, body mass index; PRA, plasma renin activity; RAS, renin-angiotensin system.

The Journal of Clinical Investigation, Inc.

Volume 95, March 1995, 948-953 ing level has been positively related to blood pressure $(2,3)$. The angiotensinogen gene ( $A G T$ ) was linked recently to human hypertension in a study from France and the United States (4). In case-control studies, two $A G T$ alleles, one that results in substitution of threonine for methionine at amino acid position 235 (T235) and one where there is substitution of methionine for threonine at position 174 (M174), were associated with hypertension in one study (4) but not in another (5). Furthermore, the serum angiotensinogen level was shown to be higher in subjects carrying the T235 allele (4).

Hypertension is more prevalent in blacks than in whites (6, 7 ), and in a previous study we showed that the blood pressure was higher and increased faster over time in black children than in white children (8). Thus, factors that contribute to the racial difference in blood pressure may be detectable in childhood. Recently, T235 was reported to be the predominant $A G T$ allele in blacks (9). Conceivably, the T235 allele and angiotensinogen contribute to the higher blood pressure of blacks. We report here on a cross-sectional study of white and black children in whom the angiotensinogen level was measured and genotyping for $A G T$ alleles was performed. We compared frequencies of alleles and the levels of angiotensinogen in the two racial groups. Since the subjects were from a longitudinal blood pressure study, we also examined the relationships of $A G T$ alleles to the longitudinal blood pressure and to the serum angiotensinogen concentration.

\section{Methods}

Subjects. Subjects were recruited from an ongoing longitudinal blood pressure study in children (8). Subjects in the original longitudinal study were recruited from 20 different schools in Indianapolis to achieve selection from a diverse socioeconomic background. The study was approved by the Institutional Review Board of Indiana UniversityPurdue University of Indianapolis. Informed consent was obtained from each child and also from his or her parents or legal guardian. Children with a history of hypertension, renal disease, cardiac disease, or diabetes mellitus and those taking medication (including birth control pills) that could affect blood pressure or the renin-angiotensin system (RAS) were excluded from the study. Subjects volunteered for the present study by providing a blood sample for isolation of DNA and plasma measurements of renin activity, aldosterone, and angiotensinogen. To determine whether those who volunteered for this study were representative of the original cohort, we compared the characteristics of age, weight, height, body mass index (BMI), sex, and race for those who participated with those who did not $(n=106)$. The means for age, weight, height, BMI, sex, and race were not significantly different between groups (all $P$ values $>0.3$ ) indicating that the sample analyzed was representative of the longitudinal study population. In the original group of subjects, there were 23 who had at least one sibling also participating in the study. To preserve the statistical independence among subjects, one sibling from each family was randomly selected for the statistical analysis. We report results for 213 subjects. Three black subjects were not 
genotyped, thus, associations with genotyping were done for a total of 210 subjects.

Measurements. Weight and height were measured at the time blood samples were drawn. In addition, because these subjects were participants from an ongoing longitudinal study, their longitudinal blood pressures during the previous $5 \mathrm{yr}$ (measured twice annually) were used in the analyses. Blood pressure was measured in the right arm with a random zero sphygmomanometer (Hawksley and Sons, Lansing, Suffex, United Kingdom) while the subject was seated. The first and fifth Korotkoff sounds were used to designate systolic and diastolic blood pressures, respectively. Three blood pressure readings were obtained, and the average of the last two readings was used as the final blood pressure measurement.

Detection of molecular alleles. Whole blood was collected in EDTAcontaining Vacutainer tubes. DNA was extracted from the white blood cells according to a standard procedure (10). DNA concentration was determined from the optical density at $260 \mathrm{~nm}$. Allele specific oligonucleotide hybridization was used for the genotyping of angiotensinogen codons 174 and 235. Genomic DNA was subjected to 30 rounds of amplification using primers 5'-GATGCGCACAAGGTCCTGTC-3' and 5'-GCCTGACTGGCTGATCTCAG-3' with 1 min denaturation at $95^{\circ} \mathrm{C}, 1 \mathrm{~min}$ annealing at $60^{\circ} \mathrm{C}$, and $1 \mathrm{~min}$ extension at $72^{\circ} \mathrm{C}$. The resulting 354-base pair fragment was denatured with $0.4 \mathrm{M} \mathrm{NaOH}, 25$ mM EDTA and dot blotted in duplicate onto nitrocellulose and then neutralized with $20 \times$ SSC. The filters were subsequently hybridized to the appropriate ${ }^{32} \mathrm{P}$-labeled oligonucleotide in a solution containing $6 \times$ SSC, $5 \times$ Denhardt's, $0.5 \%$ SDS, $0.1 \mathrm{mg} / \mathrm{ml}$ denatured salmon sperm DNA for $12 \mathrm{~h}$ at $42^{\circ} \mathrm{C}$. Filters were washed in $2 \times \mathrm{SSC}$ at the appropriate temperature for each probe.

Assay procedures. Aldosterone concentration was measured by radioimmunoassay with antiserum from Diagnostic Products Corp. (Los Angeles, CA). Plasma renin activity (PRA) was measured with a Clinical Assays GammaCoat radioimmunoassay kit (Baxter Healthcare, Cambridge, MA).

Angiotensinogen was measured as described previously (11). This was a two step procedure consisting of conversion of angiotensinogen to AI by human renin followed by the measurement of AI by radioimmunoassay (RIA). To eliminate protease activity during the first step, the incubation buffer ( $0.6 \mathrm{M}$ sodium phosphate, $36 \mathrm{mM}$ EDTA, gelatin [1 $\mathrm{mg} / \mathrm{ml}], \mathrm{pH} 6.0$ ) was brought to a boil and then slowly cooled to room temperature. Human renin was prepared as described by Haas et al. (12). For the conversion of angiotensinogen to AI, a mixture containing $0.25 \mathrm{ml}$ of the above buffer, angiotensinogen $(0.8-10 \mathrm{ng})$ or serum, $20 \mu \mathrm{l}$ of human renin, and water to $1.0 \mathrm{ml}$ was incubated at $37^{\circ} \mathrm{C}$ for $3 \mathrm{~h}$. Aliquots were then removed for measurement of the generated $\mathrm{AI}$ by RIA as described by Haber et al. (13) except for the inclusion of gelatin (Sigma grade 1) at a concentration of $1.0 \mathrm{mg} / \mathrm{ml}$ as the carrier protein in the RIA incubation buffer. The AI standard was purchased from Peninsula Labs (Taylor Way, Belmont, CA) and quantified by amino acid analysis. As a control for background, an aliquot of an incubation blank containing renin but no angiotensinogen was included in each RIA tube comprising the standard curve. A control plasma sample was assayed in each run. The angiotensinogen concentration was expressed as nanograms of AI per milliliter. The intraassay coefficient of variation for this assay was $5.8 \%(n=24)$, and the interassay coefficient of variation was $12 \%(n=81)$. The mean recovery of $\mathrm{AI}(8 \mathrm{or} 4 \mathrm{ng})$ added to the assay of control plasma samples at the start of the incubation was $92 \%$ (range, $86-101 \% ; n=4$ ).

Statistical methods. Statistical analyses of the group means for characteristics of white and black children of each gender group were compared using the $t$ test. To determine whether the angiotensinogen level was related to race and the presence of the T235 and M174 alleles, analysis of variance methods were used. Since angiotensinogen was associated with BMI, the race effect and the genotype effect were evaluated by including BMI as a covariate in the regression model. Multiple comparisons procedures were used to compare the angiotensinogen levels within each race. Statistical analyses of the relationship between blood pressure and angiotensinogen were performed using the longitudinal blood pressure measurements. For each individual the mean systolic and diastolic blood pressure were calculated. These means were modeled in terms of race, BMI, and angiotensinogen level using a general linear model. Since some individuals had more measurements than others, the analysis was weighted by the inverse of the number of visits. Statistical methods for longitudinal data suggested by Liang and Zeger (14) were also used to determine the effect of genotype and angiotensinogen level on blood pressure. The BMI was included in the model as a timedependent covariate. This analysis gave similar results to the means analysis.

\section{Results}

Characteristics of the subjects. There were 148 white and 65 black children that participated in the study. For both male and female groups, the average age and height were not significantly different between whites and blacks (Table I). Although the age distributions for whites and blacks were similar (for whites, 10.7-18.7 yr in males and 8.9-19.5 yr in females; for blacks, 9.1-18.9 yr in males and 12.3-18.1 yr in females), black children were on average $9 \mathrm{~kg}$ heavier ( $P=0.03$ for females and $P=0.05$ for males ) and had on average about a $4 \mathrm{~kg} / \mathrm{M}^{2}$ higher BMI ( $P=0.003$ for males and $P=0.005$ for females) than white children. For males, the mean longitudinal systolic blood pressure was not significantly different between whites and blacks $(P=0.58)$, but the mean longitudinal diastolic blood pressure was on average $4 \mathrm{mmHg}$ higher in blacks $(P=0.04)$. For females, both systolic and diastolic mean longitudinal blood pressures were on average $5 \mathrm{mmHg}$ higher in blacks than in whites $(P=0.05$ for the systolic and $P=0.003$ for the diastolic).

Plasma measurements of renin activity and aldosterone. PRA was not significantly different in white and black children (Table I), whereas for both males and females, plasma aldosterone was $\sim 30 \%$ lower in blacks than in whites $(P=0.02$ for males and $P=0.01$ for females). A lower plasma aldosterone level in black children has been observed previously in a population that included members of the present cohort $(15,16)$. Although PRA and plasma aldosterone were related $(r=0.32$, $P=0.0001$ ), there was no significant relationship between these variables and serum angiotensinogen (for PRA, $r$ $=-0.09, P=0.18$, and for aldosterone, $r=-0.09, P=0.20$ ).

Frequencies of the T235 and M174 alleles. The frequencies of both the M174 allele and the T235 allele were compared in white and black subjects. The frequency of the M174 allele was not significantly different in the two racial groups, 0.11 and 0.08 in whites and blacks, respectively $\left(\chi^{2}=0.55, P=0.46\right)$. On the other hand, the T235 allele was the predominant allele in blacks, occurring with a frequency of 0.81 compared with a frequency of 0.41 in whites $\left(\chi^{2}=54.9, P<0.0001\right)$. The same result was observed for either sex. The distribution of the genotypes with the T235 allele was also significantly different between the racial groups: $65 \%$ of the black children were homozygous for the T235 allele while only $18 \%$ of the white children were homozygous for this allele. The distribution of the M174 genotype was the same for whites and blacks. The distributions of genotypes for both alleles were consistent with the populations being in Hardy-Weinberg equilibrium.

Serum angiotensinogen in relation to race and genotype. For both males and females, the mean angiotensinogen level was $19 \%$ higher in black children than in white children $(P$ 


\begin{tabular}{|c|c|c|c|c|c|c|}
\hline \multirow[b]{2}{*}{ Characteristic } & \multicolumn{3}{|c|}{ Males } & \multicolumn{3}{|c|}{ Females } \\
\hline & White & Black & $P$ value & White & Black & $P$ value \\
\hline$n$ & $n=79$ & $n=32$ & & $n=69$ & $n=33$ & \\
\hline Age $(y r)$ & $14.95 \pm 1.81$ & $14.60 \pm 2.24$ & 0.39 & $14.93 \pm 2.00$ & $14.78 \pm 1.63$ & 0.70 \\
\hline Weight (kg) & $61.45 \pm 12.81$ & $70.64 \pm 21.03$ & 0.026 & $55.49 \pm 11.33$ & $64.68 \pm 16.03$ & 0.005 \\
\hline Height $(\mathrm{cm})$ & $169.13 \pm 12.99$ & $167.30 \pm 14.67$ & 0.52 & $161.21 \pm 7.90$ & $159.22 \pm 5.03$ & 0.13 \\
\hline BMI $\left(\mathrm{kg} / \mathrm{M}^{2}\right)$ & $21.4 \pm 3.4$ & $25.0 \pm 5.8$ & 0.003 & $21.2 \pm 3.4$ & $25.0 \pm 6.0$ & 0.0005 \\
\hline $\begin{array}{l}\text { Systolic blood pressure } \\
(\mathrm{mmHg})^{\ddagger}\end{array}$ & $109.14 \pm 12.08$ & $110.50 \pm 10.65$ & 0.58 & $104.32 \pm 10.67$ & $109.03 \pm 11.56$ & 0.05 \\
\hline $\begin{array}{l}\text { Diastolic blood pressure } \\
\qquad(\mathrm{mmHg})^{\ddagger}\end{array}$ & $66.11 \pm 9.04$ & $70.22 \pm 9.85$ & 0.04 & $63.97 \pm 10.10$ & $69.24 \pm 6.97$ & 0.003 \\
\hline $\log$ PRA (ng AI/ml/h) & $\begin{array}{c}1.07 \pm 0.63 \\
(3.03)^{8}\end{array}$ & $\begin{array}{c}1.05 \pm 0.57 \\
(2.56)\end{array}$ & 0.87 & $\begin{array}{c}1.05 \pm 0.60 \\
(2.76)\end{array}$ & $\begin{array}{c}0.87 \pm 0.74 \\
(2.55)\end{array}$ & 0.18 \\
\hline Plasma aldosterone (ng/dl) & $12.06 \pm 6.72$ & $8.74 \pm 6.05$ & 0.020 & $13.81 \pm 1.09$ & $9.26 \pm 7.58$ & 0.014 \\
\hline $\begin{array}{l}\text { Serum angiotensinogen } \\
\text { (ng } \mathrm{Al} / \mathrm{ml} \text { ) }\end{array}$ & $1477.1 \pm 313.1$ & $1760.8 \pm 373.8$ & 0.0001 & $1547.5 \pm 329.0$ & $1831.5 \pm 405.8$ & 0.0004 \\
\hline
\end{tabular}

* Values are means $\pm \mathrm{SD} ;{ }^{\ddagger}$ the means of longitudinal blood pressures; ${ }^{\S}$ median values without $\log$ transformation.

$=0.0001$ for males, $P=0.004$ for females) (Table I). Angiotensinogen levels were, however, highly correlated with age ( $r$ $=0.17, P=0.01)$, weight $(r=0.35, P=0.0001)$, and BMI ( $r=0.37, P=0.0001$ ). After adjusting for BMI, blacks still had on average an $11 \%$ higher angiotensinogen level than whites ( $P=0.009$ for males, $P=0.03$ for females).

To determine whether the presence of the T235 allele and race were related to the angiotensinogen level, an analysis of variance model was used. Both genotype $(P=0.01)$ and race $(P=0.0003)$ were significantly associated with the serum angiotensinogen level. The angiotensinogen levels of the three groups defined by the $A G T$ codon 235 alleles for both whites and blacks are shown in Fig. 1. Since body weight was highly correlated with the angiotensinogen level, the same analysis was carried out after adjusting for BMI; the results are presented in Table II. The effects of both genotype $(P=0.02)$ and race $(P=0.05)$ remained significant. A subanalysis for white and black children revealed that in white children the mean serum angiotensinogen level was higher in the presence of the T235 allele (Fig. 1 and Table II), whereas for black children the angiotensinogen level did not vary with genotype. This observation was consistent for the weight-adjusted angiotensinogen level as well as for the unadjusted value. The M174 allele did not show a significant association with the angiotensinogen level in either racial group.

Blood pressure in relation to genotype. To assess the relationship of the genotype with blood pressure, both the means of the longitudinal blood pressure and the changes in blood pressure over time as determined from multiple individual measurements were used. Systolic blood pressure values showed a significant relationship with genotype $(P=0.03)$. The mean systolic blood pressures for MT and MM genotype groups were, respectively, 2 and $4 \mathrm{mmHg}$ higher in comparison with the MM genotype group for both whites and blacks. Similarly, the relationship of genotype to the change in systolic blood pressure over time was significant $(P=0.04)$. The systolic blood pressures versus age for the MT and TT genotype groups were,

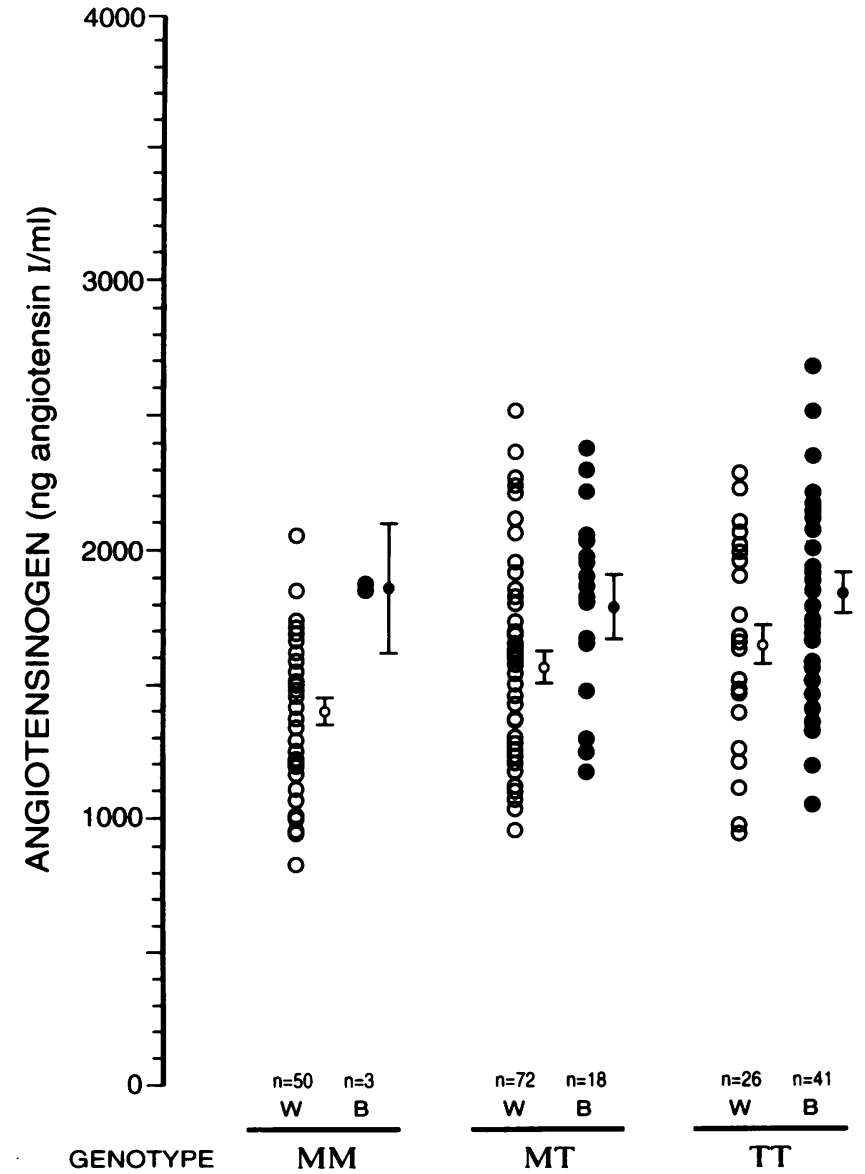

Figure 1. The serum angiotensinogen concentration in relation to genotype in white (open circles) and black (closed circles) children. M refers to the M235 allele, and T refers to the T235 allele. 
Table II. The Serum Angiotensinogen Concentration (Adjusted for BMI) in Relation to Genotype in White and Black Children*

\begin{tabular}{ccccc}
\hline & \multicolumn{4}{c}{ Angiotensinogen (ng angiotensin $\mathrm{I} / \mathrm{ml}$ ) } \\
\cline { 2 - 5 } Group & $\mathrm{MM}^{\ddagger}$ & $\mathrm{MT}^{\xi}$ & TT & Significance \\
\hline Whites $(n)$ & $1438 \pm 46(50)$ & $1577 \pm 38(72)$ & $1646 \pm 64(26)$ & $P=0.004^{\|}$ \\
Blacks $(n)$ & $1770 \pm 189(3)$ & $1685 \pm 77(18)$ & $1726 \pm 51(41)$ & $P=0.84^{\|}$
\end{tabular}

* Values are means \pm SE. ${ }^{\ddagger}$ The M235 allele; the T235 allele; " $P$ values are based on one-way analysis of variance for each race. Race effect $(P=0.05)$ and genotype effect $(P=0.02)$ were significant based on two-way analysis of variance; the interaction was not significant.

respectively, 1.8 and $4.0 \mathrm{mmHg}$ higher than for the MM genotype group. Neither the mean longitudinal diastolic blood pressure nor the change in diastolic blood pressure over time was significantly related to genotype. When the variation in blood pressure due to BMI was accounted for, the relationship of genotype to blood pressure was no longer significant with either analysis.

Blood pressure in relation to serum angiotensinogen. For both racial groups, the mean of the longitudinal diastolic blood pressure was significantly related to the angiotensinogen level with $r=0.24(P=0.0034)$ and $r=0.45(P=0.0002)$ in whites and blacks, respectively. Only black subjects showed a significant relationship to the mean of the longitudinal systolic blood pressures with $r=0.44(P=0.0003)$. After accounting for the variation in blood pressure due to BMI, the angiotensinogen level was no longer significantly related to systolic blood pressure $(P=0.19)$, but a marginally significant relationship to diastolic blood pressure remained in both whites and blacks $(P=0.07)$. The relationship between the change in blood pressure over time and the serum angiotensinogen was also significant before adjusting for BMI but not after the adjustment.

\section{Discussion}

In this study, the T235 allele of $A G T$ was the predominant allele in the black children, $81 \%$ of the $A G T$ alleles in the blacks and $42 \%$ in the whites were the T235. These findings were consistent with an earlier study in adults by Lifton et al. (9). In addition, we found the serum level of angiotensinogen to be significantly higher in black children than in white children. It is unlikely that this difference in the angiotensinogen level was an artifact of the angiotensinogen assay created by the amino acid substitution. Our assay measured generation of angiotensin I, and the kinetics for cleavage by renin of both molecular forms of angiotensinogen has been found to be identical (Lalouel, J.-M., personal communication). For the overall group, the angiotensinogen level was higher in subjects who carried the T235 allele, but within racial groups this relationship of genotype with level of angiotensinogen was significant only for white subjects.

Genes of American blacks consist of an admixture of genes of white origin. White genetic admixture within the black population in the United States has been estimated at 22\% (17) and $26 \%$ (18) in two separate studies, whereas admixture of black genes among American whites is considered to be $<1 \%$ (19). Self-reporting for race was used to assign race status in the present study; no genetic markers for race were used. We assume that the racial admixture in our subjects was similar to the earlier estimates. It is possible that the racial difference in the level of serum angiotensinogen observed in the present study may underestimate a racial difference for populations where there is no admixture among racial groups.

In previous studies in white adults, the M174 allele was in linkage disequilibrium with the T235 allele (4), and thus, although M174 was associated with hypertension (4), it was not an independent predisposing factor. In this study, the frequencies of M174 were similar in whites and blacks (0.10 and 0.08 , respectively), and no relationship of M174 to the angiotensinogen level or blood pressure was observed.

Participation in this study was restricted to individuals who had volunteered from a longitudinal study of blood pressure. A limitation imposed by this cohort was the small number of black children with the M235 allele. Thus, the lack of a relationship between the angiotensinogen level and genotype in blacks may have resulted from the small number of black children in both the groups homozygous for the M235 allele and the heterozygotes.

The relationship of angiotensinogen to blood pressure was first recognized 25 yr ago when Fasola et al. (2) found that the serum angiotensinogen concentration was higher in a group of hypertensive subjects and their offspring than in a group of normotensive subjects. Walker et al. (3) subsequently showed in a population that included hypertensives that the diastolic blood pressure was more highly related to the angiotensinogen level $(r=0.39)$ than any other parameter of blood pressure regulation analyzed. In our study of normotensive children, the angiotensinogen level showed a marginally significant relationship ( $P=0.07$ ) to diastolic blood pressure adjusted for BMI. There has been a renewed interest in angiotensinogen as a contributor to high blood pressure since it was shown that $A G T$ was linked to essential hypertension $(4,5)$ and the T235 and M174 AGT alleles were shown to occur more frequently in hypertensive populations from the United States, France (4), and Japan (20), although not in hypertensives from the United Kingdom (5). Furthermore, the frequency of the T235 allele was shown to be higher in women with preeclampsia $(21,22)$.

In this study, we observed unexpectedly that the serum angiotensinogen level was significantly related to BMI. How body size might affect the level of serum angiotensinogen is unclear, although several mechanisms can be proposed. Adipose tissue has the capacity to secrete angiotensinogen (23), and thus a higher serum angiotensinogen level might result from an increase in body fat. Insulin increases $A G T$ transcription (24) and the synthesis of angiotensinogen in vitro (25). A higher BMI can be associated with insulin resistance and hyperinsulinemia which in turn might then stimulate synthesis of angiotensinogen. It may simply be that a greater body size is required to fully express the effect of angiotensinogen on blood pressure. Alternatively, another factor, related to the age of a growing child, may increase the angiotensinogen level, such as a hormone associated with puberty or the adrenarche. Estrogen, for example, is known to increase angiotensinogen synthesis $(26,27)$. The relationship of the angiotensinogen level to age, however, was not as strong as the relationship to body size, suggesting that something unassociated with age was providing the major influence.

In addition to the effect of the T235 allele and BMI on 
serum angiotensinogen, we found evidence for an independent effect of race which resulted in a higher angiotensinogen level in blacks. This may have been related to a racial difference in regulation by $A G T$ or by differences in environmental factors. We have recently found a racial difference in the genetic regulation of the serum concentration of angiotensin I-converting enzyme (Bloem, L. J., A. K. Manatunga, E. A. Hollerman, J. H. Pratt, manuscript submitted for publication), another component of the renin-angiotensin system.

Our earlier studies in children found urinary aldosterone excretion rates to be lower in blacks than in whites $(15,16)$, and in this study the plasma aldosterone was lower in the black children. These findings are consistent with greater accumulation of sodium that suppresses the renin-angiotensin-aldosterone axis in black children. Similar evidence for sodium retention has been observed in adults. Black hypertensives typically have lower PRA (28-30) and a blood pressure that is more likely to be salt sensitive $(31,32)$ and responsive to diuretic therapy (33) when compared with white hypertensives. Conceivably, a higher angiotensinogen level initiates an increase in the kidney's production of AII which then promotes sodium retention (34). Ultimately the RAS would be down-regulated, but a persistently higher angiotensinogen level might sustain RAS activity at a level inappropriate for the degree of sodium retention. Since the angiotensinogen concentration is at the Michaelis-Menton constant for its interaction with renin (1), a higher angiotensinogen level might easily increase AII production despite lower renin activity. Secretion of aldosterone appeared to reflect a decrease more than an increase in AII. Several explanations for the lower aldosterone secretion in blacks can be proposed: $(a)$ AII may be lower in blacks than in whites but at the same time higher than physiologically appropriate for the level of accumulated sodium; $(b)$ the expression of $A G T$ may be greater in kidney than in liver where most circulating angiotensinogen originates; or $(c)$ there may be different types and degrees of responsiveness to AII in the kidney and the adrenal. In regard to the latter, the aldosterone secretory response to AII is related to the prevailing potassium concentration $(35,36)$, and blacks, in comparison with whites, may consume a diet lower in potassium, thereby reducing the effect of AII on aldosterone secretion $(37,38)$. Alternatively, a higher angiotensinogen level in blacks may not contribute to sodium retention as much as result from it. Suppression of renin activity by an accumulation of sodium could limit the catabolism of angiotensinogen by renin.

In summary, the T235 allele of $A G T$ that has been associated with hypertension in adult populations was the predominant $A G T$ allele in the black children of this study. The serum level of angiotensinogen was significantly higher in the black children than in the white children and showed a marginally significant relationship to blood pressure. These results suggest that genetic differences in the RAS may contribute to racial differences in blood pressure regulation in normal children.

\section{Acknowledgments}

The authors are grateful to Mary Anne Wagner and Dr. Chunlu Guo for technical assistance in the measurements of subjects; and to Eleanor A. Holleman for biostatistical support.

This study was supported by grants from the National Institutes of Health (RO1-HL35795 and MO1-RR00750), the Veterans Administration, and the American Heart Association (D. A. Tewksbury), and the American Heart Association Wisconsin Affiliate (D. A. Tewksbury).

\section{References}

1. Gould, A. B., and B. Green. 1971. Kinetics of the human renin and human renin substrate reaction. Cardiovasc. Res. 5:86-89.

2. Fasola, A. F., B. L. Martz, and O. M. Helmer. 1968. Plasma renin activity during supine exercise in offspring of hypertensive parents. J. Appl. Physiol. 25:410-415.

3. Walker, W. G., P. K. Welton, H. Saito, R. P. Russell, and J. Hermann. 1979. Relation between blood pressure and renin, renin substrate, angiotensin II, aldosterone and urinary sodium and potassium in 574 ambulatory subjects. Hypertension (Dallas). 1:287-291.

4. Jeunemaitre, X., F. Soubrier, Y. V. Kotelevtev, R. P. Lifton, C. S. Williams, A. Charru, S. C. Hunt, P. N. Hopkins, R. R. Williams, J.-M. Lalouel, and P. Corvol. 1992. Molecular basis of human hypertension: role of angiotensinogen. Cell. 71:169-180.

5. Caulfield, M., P. Lavender, M. Farrall, P. Munroe, M. Lawson, P. Turner, and A. Clark. 1994. Linkage of the angiotensinogen gene to essential hypertension. N. Engl. J. Med. 330:1629-1633.

6. Anonymous. 1966. Hypertension and hypertensive heart disease in adults: United States-1960-1962. Vital and Health Statistics. 11:1-62.

7. Anonymous. 1975. National Health Survey. Blood pressure of persons 18 74 years in the United States, 1971-1972. National Center for Health Statistics, Hyattsville, MD. Vital and Health Statistics; Series, 2; No. 150).

8. Manatunga, A. K., J. J. Jones, and J. H. Pratt. 1993. Longitudinal assessment of blood pressures in black and white children. Hypertension (Dallas). 22:8489.

9. Lifton, R. P., D. Warnock, R. T. Acton, L. Harman, and J. M. Lalouel. 1993. High prevalence of hypertension-associated angiotensinogen variant T235 in African Americans. Clin. Res. 41:260A.

10. Madisen, L., D. I. Hoar, C. D. Holroyd, M. Crisp, and M. E. Hodes. 1994. DNA banking: the effects of storage of blood and isolated DNA on the integrity of DNA. Am. J. Med. Genet. 27:379-390.

11. Tewksbury, D. A., E. S. Tryon, R. E. Burill, and R. A. Dart. 1986. High molecular weight angiotensinogen: a pregnancy associated protein. Clin. Chim. Acta. 158:7-12.

12. Haas, E., H. Goldblatt, E. Gipson, and L. Lewis. 1966. Extraction, purification and assay of human renin free of angiotensinase. Circ. Res. 19:739-749.

13. Haber, E., T. Koerner, L. B. Page, B. Kliman, and A. Purnode. 1969. Application of a radioimmunoassay for angiotensin I to the physiologic measurements of plasma renin activity in normal human subjects. J. Clin. Endocrinol. \& Metab. 29:1349-1355.

14. Liang, K. Y., and S. L. Zeger. 1986. Longitudinal data analysis using generalized linear models. Biometrika. 73:13-22.

15. Pratt, J. H., J. J. Jones, J. Z. Miller, M. A. Wagner, and N. S. Fineberg 1989. Racial differences in aldosterone excretion and plasma aldosterone concentrations in children. N. Engl. J. Med. 321:1152-1157.

16. Pratt, J. H., A. K. Manatunga, L. J. Bloem, and L. Wei. 1993. Racia differences in aldosterone excretion: a longitudinal study in children. J. Clin. Endocrinol. \& Metab. 77:1512-1515.

17. Reed, T. E. 1994. Caucasian genes in American Negroes. Science (Wash. DC). $165: 762-768$.

18. Chakraborty, R., M. I. Kamboh, M. Nwankwo, and R. E. Ferrell. 1992 Caucasian genes in American blacks: new data. Am. J. Hum. Genet. 50:145-155.

19. Reed, T. E. 1969. Letter to editor. Science (Wash. DC). 166:1353.

20. Hata, A., C. Namikawa, M. Sasaki, K. Sato, T. Nakamura, K. Tamura, and J.-M. Lalouel. 1994. Angiotensinogen as a risk factor for essential hypertension in Japan. J. Clin. Invest. 93:1285-1287.

21. Oppong, S. Y., and N. M. Hooper. 1993. Characterization of a secretase activity which releases angiotensin-converting enzyme from the membrane. Biochem. J. 292:597-603.

22. Arngrimsson, R., S. Purandare, M. Connor, J. J. Walker, S. Bjornsson, F. Soubrier, Y. V. Kotelevtsev, R. T. Geirsson, and H. Bjornsson. 1993. Angiotensinogen: a candidate gene involved in preeclampsia? Nature Genetics. 4:114115 .

23. Cassis, L. A., J. A. Saye, and M. J. Peach. 1988. Location and regulation of rat angiotensinogen messenger RNA. Hypertension (Dallas). 11:591-596.

24. Cassis, L. A. 1992. Downregulation of the renin-angiotensin system in streptozotocin-diabetic rats. Am. J. Physiol. 262:E105-E109.

25. Murakami, E., K. Hiwada, and T. Kokubu. 1980. Effects of insulin and glucagon on production of renin substrate by the isolated rat liver. J. Endocrinol. 85:151-153.

26. Krakoff, L. R. 1973. Measurement of plasma renin substrate by radioimmunoassay of angiotensin I: concentration in syndromes associated with steroid excess. J. Clin. Endocrinol. \& Metab. 37:110-117.

27. Clauser, E., J. Bouhnik, E. Coezy, P. Corvol, and J. Menard. 1983. Synthesis and release of immunoreactive angiotensinogen by rat liver slices. Endocrinology. 112:1188-1193. 
28. Channick, B. J., E. V. Adlin, and A. D. Marks. 1969. Suppressed plasma renin activity in hypertension. Arch. Intern. Med. 123:131-140.

29. Brunner, H. R., J. H. Laragh, and L. Baer. 1972. Essential hypertension renin and aldosterone, heart attack and stroke. N. Engl. J. Med. 286:441-449.

30. Kaplan, N. M., D. C. Kem, O. B. Holland, N. J. Kramer, J. Higgins, and C. Gomez-Sanchez. 1976. The intravenous furosemide test: a simple way to evaluate renin responsiveness. Ann. Intern. Med. 84:639-645.

31. Luft, F. C., L. I. Rankin, R. Bloch, A. E. Weyman, L. R. Willis, R. H. Murray, C. E. Grim, and M. H. Weinberger. 1979. Cardiovascular and humora responses to extremes of sodium intake in normal black and white men. Circula tion. 60:697-706.

32. Weinberger, M. H., J. Z. Miller, F. C. Luft, C. E. Grim, and N. S. Fineberg. 1986. Definitions and characteristics of sodium sensitivity and blood pressure resistance. Hypertension (Dallas). 8(Suppl. II):II-127-II-134.

33. Materson, B. J., D. J. Reda, W. C. Cushman, B. M. Massie, E. D. Freis M. S. Kochar, R. J. Hamburger, C. Fye, R. Lakshman, J. Gottdiener, E. A Ramirez, and W. G. Henderson. 1993. Single-drug therapy for hypertension in men: a comparison of six antihypertensive agents with placebo. $N$. Engl. J. Med. 328:914-921.

34. Hall, J. E. 1986. Control of sodium excretion by angiotensin II: intrarenal mechanisms and blood pressure regulation. Am. J. Physiol. 250:R960-R972.

35. Pratt, J. H. 1982. Role of angiotensin II in potassium-mediated stimulation of aldosterone secretion in the dog. J. Clin. Invest. 70:667-672.

36. Pratt, J. H., J. K. Rothrock, and J. H. Dominquez. 1989. Evidence that angiotensin-II and potassium collaborate to increase cytosolic calcium and stimulate the secretion of aldosterone. Endocrinology. 125:2463-2469.

37. Watson, R. L., H. G. Langford, J. Abernethy, T. Y. Barnes, and M. J. Watson. 1980. Urinary electrolytes, body weight, and blood pressure: pooled cross-sectional results among four groups of adolescent females. Hypertension (Dallas). 2:93-98.

38. Grim, C. E., F. C. Luft, and J. Z. Miller. 1980. Racial differences in blood pressure in Evans County, Georgia: relationship to sodium and potassium intake and plasma renin activity. J. Chronic Dis. 33:87-94. 